\title{
Laser Microdissection and Laser Pressure Catapulting for the Generation of Chromosome- Specific Paint Probes
}

BioTechniques 27:362-367 (August 1999)

\section{Schermelleh, S. Thalham- mer, W. Heckl, H. Pösl' ${ }^{\text {, }}$ T. Cremer, K. Schütze ${ }^{1}$ and M. Cremer \\ Ludwig Maximilians Univer- sität, Munich, ${ }^{1}$ Academic Hospital München-Harlaching, Munich, Germany}

\section{INTRODUCTION}

Chromosome-specific DNA probes can be generated by isolating DNA from whole chromosomes or chromosomal subregions and its subsequent universal amplification by degenerate oligonucleotide-primed polymerase chain reaction (DOP-PCR). Such paint probes allow the direct visualization of specific chromosomes or sub-chromosomal regions in metaphase spreads as well as chromosomal territories in interphase nuclei by fluorescence in situ hybridization (FISH). The availability of such paint probes has become an important tool for cytogenetic analysis with wide applications, ranging from research studies of chromosome territories to diagnostic use in clinical genetics $(1,2)$.

Different methods are available for the collection of such samples. (i) Chromosomes isolated by flow sorting have been successfully applied for the generation of whole chromosome-specific libraries from various species, which usually originate from several hundreds of sorted chromosomes $(11,16)$. Flow sorting works best on chromosomes that have a wide range of sizes or present discrete differences in base pair composition. (ii) The conventional microdissection approach uses fine glass needles for the collection of chromosomes $(6,9,10)$ and also allows the isolation of sub- chromosomal regions of GTG (G-bands by trypsin using Giemsa)-banded chromosomes with high precision (7). However, needle dissection is tedious and needs especially skilled personnel. Furthermore, this method requires mechanical contact and therefore is sensitive to contamination.

In this paper, we present a method for microdissection and rapid collection of chromosomes that is solely based on laser micromanipulation. We demonstrate that this approach allows the fast generation of chromosome-specific paint probes originating from a few or only one copy of a chromosome without mechanical contact. The entire approach comprises $(i)$ the mounting of metaphase spreads on ultrathin membranes, (ii) the optional destruction of unwanted genetic material adjacent to the target chromosome by laser ablation, (iii) the isolation of the separated chromosomes by laser dissection of the membrane around the target chromosome, (iv) the collection of the chromosome-membrane stack by laserpressure catapulting (LPC) onto a collection device and (v) universal DNA amplification of the collected chromosomes by DOP-PCR and labeling of the amplified probe.

The chromosomal specificity of the obtained probes was demonstrated by hybridizing the labeled probes to normal metaphases. 


\section{MATERIALS AND METHODS}

\section{Materials}

Metaphase chromosomes were obtained from cell cultures of a diploid kidney cell line from Chinese hamster (kindly provided by S. Müller, University of Munich, Germany) and from short-term cultures of human lymphocytes according to standard procedures.

\section{Metaphase Chromosome Spreads}

A piece of a $2 \times 3$-cm-sized, 1.35$\mu \mathrm{m}$-thick polyethylene naphthalate (PEN) or polyester-based (NYLA) membrane (provided by P.A.L.M. $\mathrm{GmbH}$, Bernried, Germany) was placed on a pre-cleaned slide, fixed with $\mathrm{Scotch}^{\circledR}$ tape and UV-irradiated $(254 \mathrm{~nm})$ for $30 \mathrm{~min}$. Mounting was facilitated by a drop of pure ethanol placed on the coverslip. Metaphase chromosome suspensions from the Chinese hamster cells or human lymphocytes fixed in 3:1 methanol:acetic acid were dropped onto the membrane. After $30 \mathrm{~s}$, the slide was dipped briefly into $70 \%$ acetic acid to obtain wellspread metaphases, then dehydrated through an alcohol series $(70 \%, 90 \%$ and $100 \%$ ) and air-dried. Giemsa staining was performed in a $0.1 \%$ Giemsa solution for $10 \mathrm{~min}$, slides were thoroughly rinsed with sterile, filtered water and air-dried. For GTG-banding, slides were aged at $56^{\circ} \mathrm{C}$ overnight and incubated in $0.1 \%$ trypsin for $20-40 \mathrm{~s}$ before Giemsa-staining.

\section{Laser Microbeam Microdissection (LMM)}

A commercially available UV-Laser Microbeam System (RoBOT-MicroBEAM; P.A.L.M. GmbH) was used as previously described (12). This system consists of a 337-nm nitrogen laser that is coupled into the light path of an inverted microscope and focused through an oil immersion objective $(63 \times$ or $100 \times$ magnification, respectively) with high numerical aperture to yield a spot size of less than $1 \mu \mathrm{m}$ in diameter (13). The microscope stage (Robot-Stage), the micromanipulator (Robot-Manipulator) and the laser micromanipulation procedure are computer-controlled. The microscope image is transferred by a video camera and displayed on a video screen. An energy of $0.2-0.3 \mu \mathrm{J} /$ pulse was used for ablation and of $0.5-0.6$ $\mu \mathrm{J} /$ pulse for membrane dissection.

\section{LPC}

For catapulting, the laser was focused slightly below the membrane. The isolated chromosome-membrane islets were ejected from the object slide with a single laser shot of $1-2 \mu \mathrm{J} /$ pulse and catapulted onto a collection device, held at a distance of $<1 \mathrm{~mm}$ above the slide by a micromanipulator as shown in Figure 1, A and B. The collecting device consists of a small $(1.5 \times 3 \mathrm{~mm})$ piece of glass heat-sealed to a pipet tip. Pure glycerol $(0.1 \mu \mathrm{L})$ was placed on the collection glass to improve the adherence of the catapulted specimen and to facilitate their release into a reaction tube. Successful collecting of the catapulted membranes into the glycerol drop was monitored by focusing an objective of low magnification onto the collecting glass.

\section{DOP-PCR}

After LPC, the glass particle was transferred into a reaction tube and centrifuged at $1000 \times g$ for $3 \mathrm{~min}$ to get the glycerol drop with the selected chromosomes into the bottom of the tube. The glass piece was then removed using a clean forceps. The amplification of the sample DNA was performed with the partially degenerate universal primer 6MW (5'-CCG ACT CGA GNN NNN NAT GTG G-3') (15) in a final volume of $25 \mu \mathrm{L}$ of a PCR mixture containing $60 \mathrm{mM}$ Tris- $\mathrm{HCl}, \mathrm{pH}$ 8.5, $15 \mathrm{mM}\left(\mathrm{NH}_{4}\right)_{2} \mathrm{SO}_{4}, 3.5 \mathrm{mM}$ $\mathrm{MgCl}_{2}, 0.05 \% \mathrm{~W}-1$-detergent (Life Technologies, Paisley, Scotland, UK), $200 \mu \mathrm{M}$ of each dNTP, $1.5 \mu \mathrm{M} 6 \mathrm{MW}$ primer and 1.5 U Taq DNA Polymerase (Life Technologies). Primary amplification was performed on a PROGENE Thermal Cycler (Techne, Princeton, NJ, USA) with the following cycling parameters: initial denaturation at $96^{\circ} \mathrm{C}$ for $3 \mathrm{~min}, 8$ low-stringency cycles of $96^{\circ} \mathrm{C}$ for $1 \mathrm{~min}, 30^{\circ} \mathrm{C}$ for $1 \mathrm{~min}, 3-\mathrm{min}$ transition of $30^{\circ}$ to $72^{\circ} \mathrm{C}$ and $72^{\circ} \mathrm{C}$ for 2 min, followed by 35 high-stringency cycles of $94^{\circ} \mathrm{C}$ for $1 \mathrm{~min}, 56^{\circ} \mathrm{C}$ for 1 $\min , 72^{\circ} \mathrm{C}$ for $2 \mathrm{~min}$ and a final extension of 5 min at $72^{\circ} \mathrm{C}$.

Two microliters of the primary amplification product were labeled with digoxigenin or biotin in a secondary-



Figure 1. Schematic diagram of the LMM/LPC procedure. 
label PCR in a volume of $50 \mu \mathrm{L}$ of a PCR mixture containing $10 \mathrm{mM}$ Tris$\mathrm{HCl}, \mathrm{pH} 8.3,2 \mathrm{mM} \mathrm{MgCl}_{2}, 50 \mathrm{mM}$ $\mathrm{KCl}, 200 \mu \mathrm{M}$ of dATP, dCTP and dGTP each, $160 \mu \mathrm{M}$ dTTP, $40 \mu \mathrm{M}$ Digoxigenin-11-dUTP or Biotin-16dUTP (Boehringer Mannheim GmbH, Mannheim, Germany), $1.5 \mu \mathrm{M} 6 \mathrm{MW}$ primer and $2 \mathrm{U}$ Taq DNA polymerase. PCR conditions were as follows: initial denaturation at $94^{\circ} \mathrm{C}$ for $3 \mathrm{~min}, 20 \mathrm{cy}$ cles of $94^{\circ} \mathrm{C}$ for $1 \mathrm{~min}, 56^{\circ} \mathrm{C}$ for $1 \mathrm{~min}$, $72^{\circ} \mathrm{C}$ for $30 \mathrm{~s}$ and final extension $72^{\circ} \mathrm{C}$ for $3 \mathrm{~min}$. The probe size, checked on a $1 \%$ agarose gel, was in the range between 300 and $800 \mathrm{bp}$.

\section{FISH}

Approximately $50 \mathrm{ng} / \mu \mathrm{L}$ of the labeled probe with $1 \mu \mathrm{g} / \mu \mathrm{L}$ of the respective Cот-1 DNA ${ }^{\circledR}$ (Life Technologies) were hybridized on metaphase chromosomes and detected according to standard procedures (8).

Photographs were taken with a Zeiss Axiophot ${ }^{\circledR}$ Epifluorescence Microscope (Carl Zeiss Jena GmbH, Jena, Germany). Digital images were obtained using a cooled charge-coupled device (CCD) camera (Photometrics, Tucson, AZ, USA) coupled to the microscope. Camera control and digital image acquisition were performed on a Cytovision system (Applied Imaging, Santa Clara, CA, USA).

\section{RESULTS AND DISCUSSION}

The LMM technique and the LPC procedure were recently described for the capture of single cells (14); however, to our knowledge, they have not yet been used for the collection of chromosomes and the generation of chromosome-specific paint probes. The LMM/ LPC technique utilizes the light-pressure force caused by the high photon density within the narrow focus of the laser beam, and it differs significantly from the "cookie-cutter" method (4) and from the recently described lasercapture-microdissection (3), where an infrared heat-generating laser is used to melt the area-of-interest onto a transfer membrane. Figure 1 schematially outlines the principle of our approach.

The morphology and Giemsa stain- ing of the metaphase chromosomes, spread on a UV-irradiated ultrathin supporting membrane, was comparable to those spread directly on glass slides (Figure 2, A and E). The laser microbeam was used to first cut the membrane around a chromosome-of-interest, yielding an islet with a diameter of 5-10 $\mu \mathrm{m}$ (Figure 2, B and G). Dissection of the membrane could be achieved using pulse energies of $0.5-0.6 \mu \mathrm{J} / \mathrm{pulse}$. Subsequently, the dissected membranes were catapulted right away onto the collection device by slightly focusing the laser below the membrane level (Figure 2, $\mathrm{C}$ and $\mathrm{H}$ ). Using an energy of $1-2 \mu \mathrm{J} /$ pulse, the majority of catapulted membrane pieces could be recovered on the collection device (Figure 2D). If numerous copies of a homologous chromosome were isolated, they were all catapulted on one collecting device.

The chromosomal material could then be transferred on the collection device to a reaction tube and released into the tube by centrifugation.

For a first evaluation of our approach, we selected well-spread chromosomes as shown for a metaphase of the Chinese hamster in Figure 2, A-D. We wanted to test whether it is possible to isolate a single chromosome by the noncontact LMM/LPC procedure without destroying the genetic information.
After optimizing the buffer conditions in the PCR, the DNA of the captured chromosomes was sufficiently accessible to serve as target DNA for a universal amplification. In case of one single copy, the amount of DNA for amplification was less than one picogram. Reverse-painting of the DOP-labeled probe on respective metaphase spreads resulted in the specific hybridization over the entire length of the captured chromosomes as shown for Chinese hamster chromosomes 1 (Figure 3A) and $\mathrm{Y}$ (Figure 3C), which shows a specific cross-hybridization of the homologous region on Xq. Thus, our results demonstrate that the laser energy used for membrane dissection, in a distance of 2-5 $\mu \mathrm{m}$ around the target chromosome and for the catapulting procedure, does not alter the target DNA, at least not to an extent that noticeably interferes with DOP-PCR amplification. This is probably due to the fact that the applied laser wavelength of $337 \mathrm{~nm}$ is sufficiently distant from the absorption maximum of DNA of $260 \mathrm{~nm}$ and that the effective laser energy is limited within a narrow focal area (5). As one copy of a chromosome is sufficient for the generation of a specific paint probe, the identification of individual chromosomes (e.g., by GTG banding before microdissection) is not absolutely necessary. This makes our approach espe-

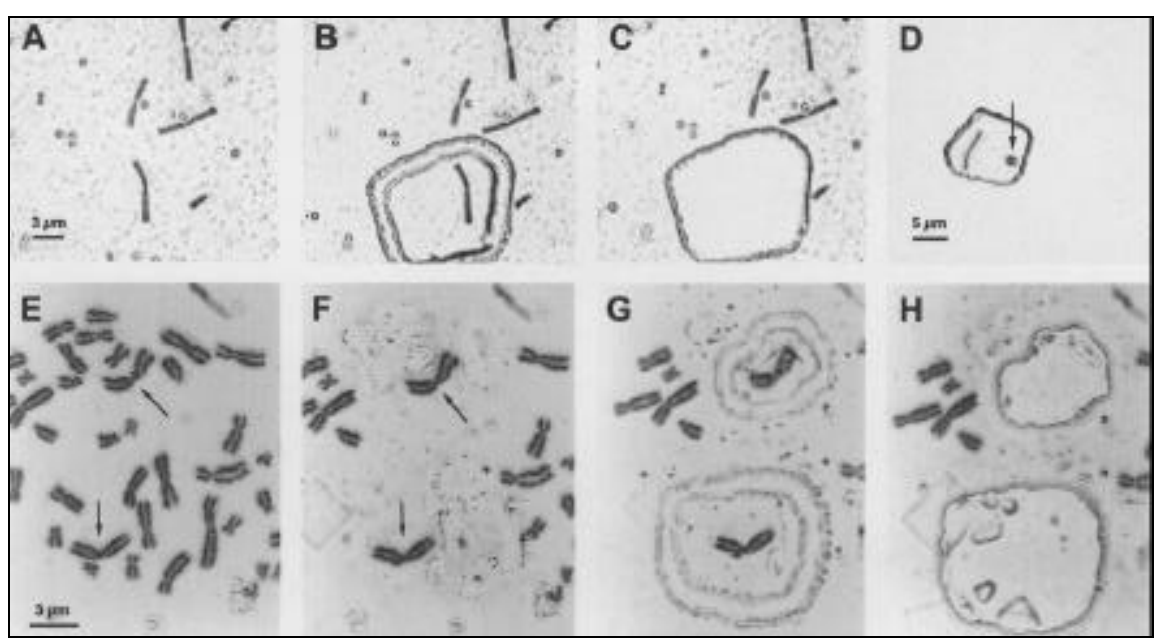

Figure 2. Isolation of metaphase chromosomes by LMM/LPC. Panels A-D show a Chinese hamster metaphase; Panels E-H show a human metaphase. (A) Chromosomes fixed on a PEN membrane; (B) isolation of hamster chromosome 1 by membrane dissection; (C) isolated region after catapulting; (D) monitoring of the catapulted chromosome-membrane stack on the collecting glass; arrow indicates spot caused by laser shot (16× magnification); (E) human metaphase chromosomes fixed on a polyester membrane; arrows indicate chromosomes 1; (F) ablation of numerous chromosomes around the chromosomes 1; (G) isolation of selected chromosomes 1 by membrane dissection; $(\mathrm{H})$ isolated regions after catapulting. 
cially attractive for chromosome-specific probe generation of species where unequivocal identification of specific chromosomes by size or banding analysis is difficult or even impossible. In such cases, to avoid the excess collection of identical chromosomes from different metaphases, the preparation of widely spread metaphases that allow the isolation of numerous chromosomes from one metaphase is highly desirable. Irrespective of this application, the identification of chromosomes by GTG banding wherever possible would be helpful for many purposes. Therefore, we have tested several conditions of banding procedures. However, the banding quality of membranemounted chromosomes was always less compared to chromosomes directly spread on glass slides and did not allow the reliable identification of all chromosomes from a membrane-mounted metaphase (data not shown).

A key feature for future applications of the LMM/LPC technique would be the ability to capture discrete subregions of a chromosome as, i.e., chro-
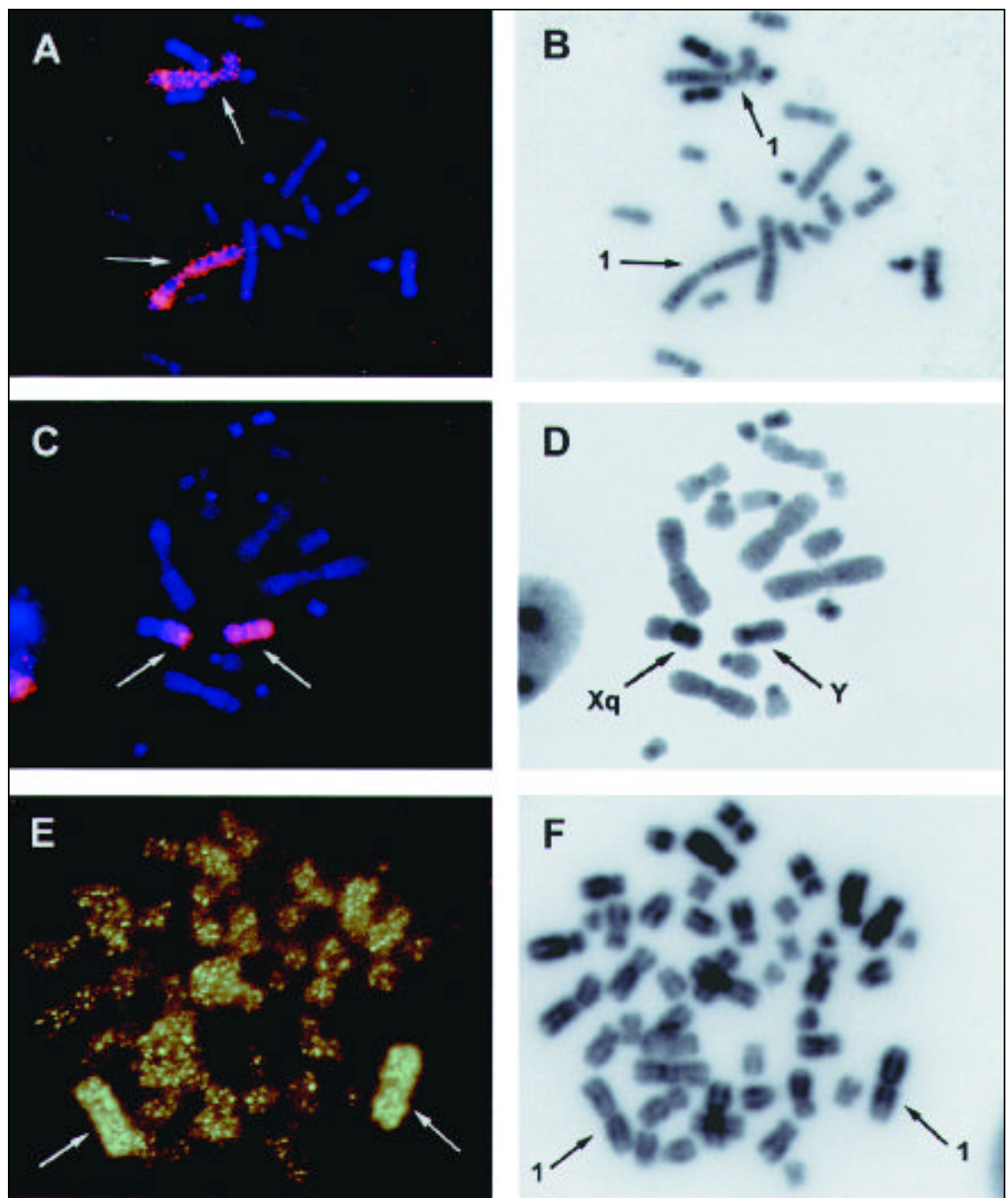

Figure 3. Hybridization of DNA probes obtained by LMM/LPC. (A) Hamster chromosome 1 probe derived from one isolated copy, detected with anti-DIG-Cy ${ }^{\mathrm{TM}} 3$ and counterstained with 4',6-diamidine2'-phenylindole dihydrochloride (DAPI); (B) Inverted DAPI picture of the same metaphase; (C) Hamster chromosome Y probe derived from one isolated copy of a GTG-banded preparation, detected with anti-DIG-Cy3 and counterstained with DAPI; note the specific cross-hybridization of the homologous region on Xq; (D) inverted DAPI picture of the same metaphase; (E) human chromosome 1 probe derived from 15 isolated copies detected with avidin-fluorescein isothiocyanate (FITC); some unspecific hybridization on other chromosomes is observed; (F) inverted DAPI picture of the same metaphase. mosome arms or finer sub-chromosomal regions. In principle, two approaches can be pursued for the isolation of such sub-chromosomal fragments. (i) A sub-chromosomal fragment could be directly isolated by cutting the underlying membrane along the respective target fragment. However, the dissection of membranes that are presently available requires a laser energy of at least $0.5 \mu \mathrm{J} /$ pulse, resulting in a cutting width of approximately $3 \mu \mathrm{m}$, including the structurally altered rim as shown in Figure 2B. Such cutting lines are not sufficiently fine for the precise isolation of an intact chromosomal subfragment. This present limitation could be overcome as soon as membranes become available that require less energy for cutting and thus will allow very fine cutting lines. (ii) Alternatively, the isolation of chromosomal subregions could be achieved by preceding ablation of unwanted genetic material adjacent to the target chromosomal fragment before dissecting the membrane 
at a wider distance. This approach could also be used for the isolation of chromosomes where well-spread metaphases could not be achieved. Figure $2 \mathrm{~F}$ shows an example of preceding laser-ablation of the chromosomes around the chromosomes 1 of a human metaphase spread.

What is questionable is whether the laser-ablation procedure destroys the ablated chromosomal material, not only structurally, but to an extent that even prevents any amplification by DOPPCR.

In our experiments, we used an energy of $0.2-0.3 \mu \mathrm{J} /$ pulse for ablation of chromosomes. As shown in Figure 2F, this energy resulted in an optical destruction of ablated DNA but still left the underlying membrane intact and did not alter the shape of the chromosomes to be isolated. DOP-PCR resulted in a specific paint of the respective chromosome, as shown in Figure $3 \mathrm{E}$ for chromosome 1 , where 15 copies had been isolated after ablation of the closely adjacent chromosomes of the respective metaphases. Thus, ablation of DNA material around the target DNA within a distance of down to 1 $\mu \mathrm{m}$ did not noticeably impair DOP amplification. However, in addition to the specific hybridization pattern, some unspecific hybridization signals on most of the chromosomes could be seen. Such an unspecific hybridization pattern was not observed using probes from sufficiently spread chromosomes, where preceding-ablation was not necessary. We assume that this unspecific hybridization is most probably due to an incomplete destruction of the ablated DNA, which, although invisible on the membrane after ablation, could still be DOP-amplified to some extent. This assumption is supported by our finding that DOP-PCR products of a catapulted membrane piece from which an entire metaphase had been visibly ablated, also resulted in some background hybridization. Contamination problems could be excluded, as amplifications from empty catapulted membrane pieces collected from the same slide did not result in any positive hybridization (data not shown).

We are currently working on improved conditions for the generation of background-free paint probes from sub-chromosomal regions. Once these conditions have been established, the combination of LMM and LPC will become a valuable method also for the generation of specific paint probes from sub-chromosomal regions.

\section{ACKNOWLEDGMENTS}

This work was supported by the Deutsche Forschungsgemeinschaft (DFG) Grant No. He 16/17 3-3 and the Bayerische Forschungsstiftung Grant No. 153/95. We also thank S. Müller (Institute of Anthropology and Human Genetics) for providing the Chinese hamster cell line and R. Schütze (P.A. L.M., Bernried) for technical support.

\section{REFERENCES}

1.Carter, N.P., M.A. Ferguson-Smith, M.T. Perryman, H. Telenius, A.H. Pelmar, M.A. Leversha, M.T. Glancy, S.L. Wood et al. 1992. Reverse chromosome painting: a method for the rapid analysis of aberrant chromosomes in clinical cytogenetics. Med. Genet. 29:299-307.

2.Cremer, T., A. Kurz, R. Zirbel, S. Dietzel, B. Rinke, E. Schröck, M.R. Speicher, U. Mathieu et al. 1993. Role of chromosome territories in the functional compartmentalization of the cell nucleus, p. 777-791. Cold Spring Harbor Symposia on Quantitative Biology, Volume VLIII. CSH Laboratory Press, Cold Spring Harbor, NY.

3.Emmert-Buck, M.R., R.F. Bonner, P.D. Smith, R.F. Chuaqui, Z. Zhuang, S.R. Goldstein, R.A. Weiss and L.A. Liotta. 1996. Laser capture microdissection. Science 274:998-1001.

4.Fukui, K., M. Minezawa, Y. Kamisugi, M. Ishikawa, N. Ohmido, T. Yanagisawa, M. Fujishita and F. Sakai. 1992. Microdissection of plant chromosomes by argon-ion laser beam. Theor. Appl. Genet. 84:787-791.

5.Greulich, K.O. and G. Leitz. 1994. Light as microsensor and micromanipularor: laser microbeams and optical tweezers. Exp. Technique Phys. 40:1-14.

6.Guan, X.Y., P.S. Meltzer and J.M. Trent. 1994. Rapid generation of whole chromosme painting probes (WCPs) by chromosome microdissection. Genomics 22:101-107.

7.Guan, X.Y., H. Zhang, M. Bittner, Y. Jiang, P. Meltzer and J.M. Trent. 1996. Chromosome arm painting probes. Nature Genet. 12:10-11.

8.Lichter, P. and T. Cremer. 1992. Chromosome analysis by non-isotopic in situ hybridization, p. 157-192. In D.E. Rooney and B.H. Czepulkowski (Eds.), Human Cytogenetics (Vol I): A Practical Approach, 2nd ed. IRL Press, Oxford, UK.

9.Lüdecke, H.J., G. Senger, U. Claussen and
B. Horsthemke. 1989. Cloning defined regions of the human genome by microdissection of banded chromosomes and enzymatic amplification. Nature 338:348-350.

10.Meltzer, P.S., X.Y. Guan, A. Burgess and J.M. Trent. 1992. Rapid generation of region specific probes by chromosome microdissection and their application. Nature Genet. 1:2428.

11.Rabbitts, P., H. Impey, A. Heppell-Parton, C. Langford, C. Tease, N. Lowe, D. Bailey, M. Ferguson-Smith and N. Carter. 1995. Chromosome specific paints from a high resolution flow karyotype of the mouse. Nature Genet. 9:369-375.

12.Schütze, K., I. Becker, K.F. Becker, S. Thalhammer, R. Stark, W.M. Heckl, M. Böhm and H. Pösl. 1997. Cut out or poke in - the key to the world of single genes: laser micromanipulation as a valuable tool on the lookout for the origin of disease. Genet. Anal. Biomol. Eng. 14:1-8.

13.Schütze, K. and A. Clement-Sengewald. 1994. Catch and move-cut and fuse. Nature 368:667-669.

14.Schütze, K. and G. Lahr. 1998. Identification of expressed genes by laser-mediated manipulation of single cells. Nature Biotechnol. 16:737-742.

15.Telenius, H., N.P. Carter, C.E. Bebb, M. Nordenskjöld, B.A.J. Ponder and A. Tunnacliffe. 1992. Degenerate oligonucleotideprimed PCR: general amplification of target DNA by a single degenerate primer. Genomics 13:718-772.

16.Telenius, H., A.H. Pelmear, A. Tunnacliffe, N.P. Carter, A. Behmel, M.A. FergusonSmith, M. Nordenskjöld, R. Pfragner and B.A.J. Ponder. 1992. Cytogenetic analysis by chromosome painting using DOP-PCR amplified flow sorted chromosomes. Genes Chromosomes Cancer 4:257-263.

Received 17 December 1998; accepted 21 April 1999.

Address correspondence to:

Dr. Marion Cremer

Institute of Anthropology and Human Genetics

Ludwig Maximilians Universität

Richard-Wagnerstre 10/1

D-80333 Munich, Germany

Internet:marion.cremer@lrz.uni-muenchen.de 